\title{
Impact Analysis of EV Charging with Mixed Control
}

\section{Strategy}

\author{
Di Wu ${ }^{1}$, Haibo Zeng ${ }^{2}$ and Benoit Boulet ${ }^{1}$ \\ 1. Department of Electric and Computer Engineering, McGill University, Montreal, QC H3A 0G4, Canada \\ 2. Department of Electric and Computer Engineering, Virginia Tech, Blacksburg, VA 24061, USA
}

Received: May 20, 2015 / Accepted: June 15, 2015 / Published: August 31, 2015.

\begin{abstract}
EVs (electric vehicles) have been widely accepted as a promising solution for reducing oil consumption, air pollution and greenhouse gas emission. The number of EVs is growing very fast over the years. However, the high adoption of EVs will impose a burden on the power system, especially for neighborhood level network. In this paper, we propose a mixed control framework for EV charging scheduling to mitigate its impact on the power network. A metric for modeling customer's satisfaction is also proposed to compare the user satisfaction for different algorithms. The impacts of the proposed algorithms on EV charging cost, EV penetration and peak power reduction are evaluated with real data for a neighborhood level network. The simulation results demonstrate the effectiveness of the proposed algorithms.
\end{abstract}

Key words: Neighborhood level network, electric vehicle, penetration level, mixed control, charging management, user satisfaction.

\section{Introduction}

With the growing concerns on environmental protection and energy security, more and more countries and regions are promoting the usages of electric vehicles. EV (electric vehicle) is globally treated as a promising alternative to conventional vehicles to reduce greenhouse gas emission, and is expected to see a fast development in the near future [1]. This growing trend of transportation electrification has been reported in a few works [2,3]. For example, it is estimated that, $23 \%$ of all the vehicles will have an alternative powertrain in 2020 [3].

On the other hand, the high adoption of EVs will also impose great challenges on the power system. EV charging power consumption is estimated to be comparable to typical base load for neighborhood level customers [4]. If the EV charging is left uncontrolled, the peak power consumption will be greatly increased, which accelerates the grid ageing.

Corresponding author: $\mathrm{Di} \mathrm{Wu}$, Ph.D. student, research fields: stochastic optimization and optimal scheduling. E-mail: di.wu5@mail.mcgill.ca.
This issue has drawn attention in the research community [5-8]. It will be even worse for neighborhood level networks, where EV adopters often charge at similar times imposing clustering load to the network $[9,10]$.

EV charging can be treated as a kind of flexible load consumption [11], as we only need to get the EVs charged with enough energy before the departure time. Several control strategies have been proposed for EV charging scheduling. There are mainly two groups of control strategies: centralized control and decentralized control strategies [12]. For the centralized control strategies, usually there is a central controller which can access all information and control all the charge decisions for all the EVs. In Ref. [8], the authors propose a centralized EV charging control method which can help minimize the total charging cost while considering the negative impacts on the grid network. In Ref. [13], charging fairness for customers is discussed in a centralized control framework.

Centralized control could make full use of the 
charging flexibilities of all the EVs to reduce the charging cost and mitigate the adverse effects on power system. However, there will be a heavy communication burden for the centralized control strategies. Besides, the owner of the EV may not necessarily be willing to give up the control to the central controller, partly due to privacy issues. Because of these concerns, not all the people adopt the centralized control strategy [14]. To overcome these shortcomings, Refs. [15-20] discuss the decentralized control strategies for EV charging which only require no or little information exchange between electric vehicles and the system controller. In Ref. [15], the charging price is used as a signal to realize the decentralized control for EVs. In Ref. [20], the authors proposed a decentralized control strategy with genetic algorithm.

Centralized control would be easier to get the global optimum as it can control the charging for all the EVs. However, decentralized control could provide the customers with more flexibility. For some specific neighborhood level networks, it could be possible that, only some of the customers are willing to accept the centralized control. To address this problem, we propose a mixed EV charging control framework which can recognize the need of decentralized control while maximizing the benefit of centralized control for both the grid companies and the customers.

Within the proposed control framework, the system operator is responsible for the charging control and price signal design. We consider two types of customers in the neighborhood level network. For the customers who are willing to give out the charging control, the charging for these EVs will be controlled by system controller. For those customers who are not willing to give out the charging control, the charging for these EVs will be influenced by time-of-use price signal. To describe the user satisfaction, a new user satisfaction model is proposed. The impacts of the proposed mixed control algorithm on the charging cost, EV penetration, and peak power reduction will be studied with simulation using real data.

The organization of this paper is organized as follows: In Section 2, the system models are described; in Section 3, the proposed mixed control framework is presented; in Section 4, the simulation results are presented with real data for a neighborhood level network; finally, the paper is concluded in Section 5.

\section{System Models}

In this Section, we describe the proposed charging control framework, including the models for EV customers, base load power consumption, electric vehicle charging power consumption and user satisfaction.

\subsection{EV Customers and Base Load Power Consumption}

Fig. 1 shows the prototype for neighborhood level network. In the neighborhood level network, there could be one or more EVs in every home. We consider that, there are two groups of customers for a given neighborhood level network: contractual and non-contractual customers. The contractual customers would like to sign the contract with the utility companies and give out the charging control of their EVs. The other group of customers would prefer to having the charging freedom and are unwilling to give out the charging control.

For the neighborhood level network integrated with EVs, we classify the power consumptions into two categories: base load power consumption and EV charging power consumption. The base load power consumption includes all the other power consumptions except the EV charging power consumption, for

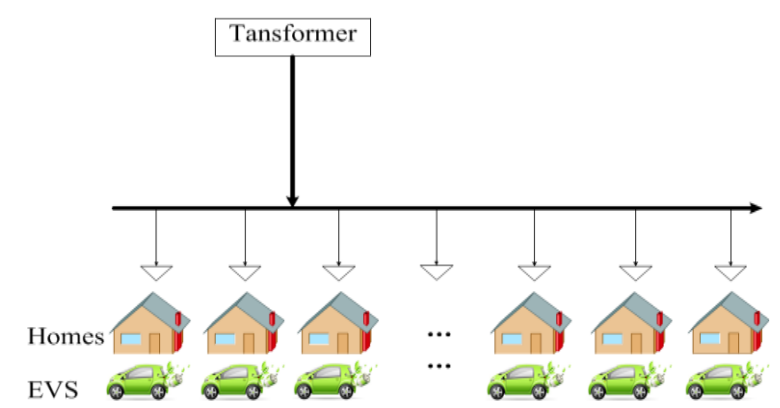

Fig. 1 Neighborhood network integrated with EVs. 
example, heating, cooling, lighting and other facility power consumptions. The base load power consumptions of a neighborhood level network with seven homes for two representative days, one in summer and the other in winter, are demonstrated in Fig. 2.

In Fig. 2, it can be seen that, the peak hours are usually around noon in summer (due to the need for cooling) and the evening in winter (for heating). For uncontrolled charging, people would start charging their EVs when they arrive home in the evening, which may overlap with the base load consumption peak hours. This will further increase the peak power consumption and accelerate the grid ageing.

Fig. 3 shows the total power for the neighborhood level network integrated with four electric vehicles. In Fig. 3, the line "total load 1" represents the total power consumption of the network integrated with four Honda Fit EVs, and the line "total load 2" gives the total power consumption integrated with four Tesla Model S EVs. These EVs are assumed to be charged continuously with their rated charging power. From Fig. 3, it is clear that, even for the network integrated with four EVs (a penetration level of 4/7), the total power consumption will be very close to the power limit for the neighborhood level network.

\subsection{Electric Vehicle Charging Load}

In this paper, we consider two kinds of EVs: Honda Fit EV and Tesla Model S EV. The specifications for these EVs are listed in Table 1 [21, 22]. Honda Fit is relatively economic and can get fully charged around $3 \mathrm{~h}$ with its maximum charging power. Tesla Model $\mathrm{S}$ has a much larger battery pack, and can get fully charged in about $6 \mathrm{~h}$. We consider continuous charging, which means that the EVs can be charged with any power from zero to maximal rated charging power.

We use the survey results conducted in City of Ottawa [23] to study the customers' driving behaviors. The results are described in Fig. 4, Tables 2 and 3.

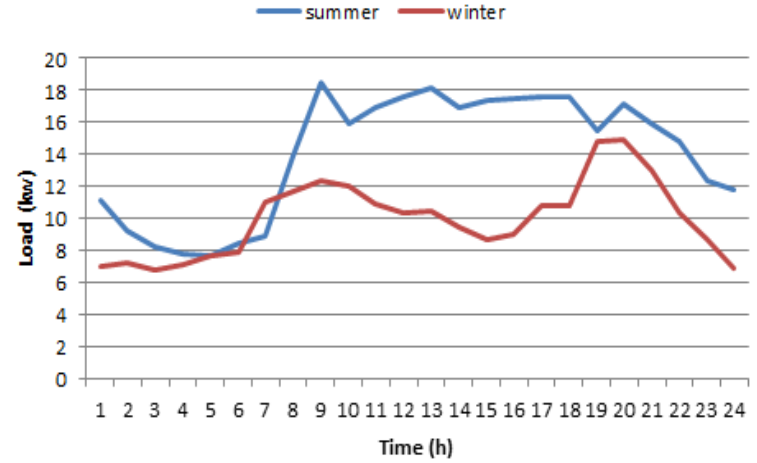

Fig. 2 Base load consumption for a neighborhood network with seven homes in two representative days.

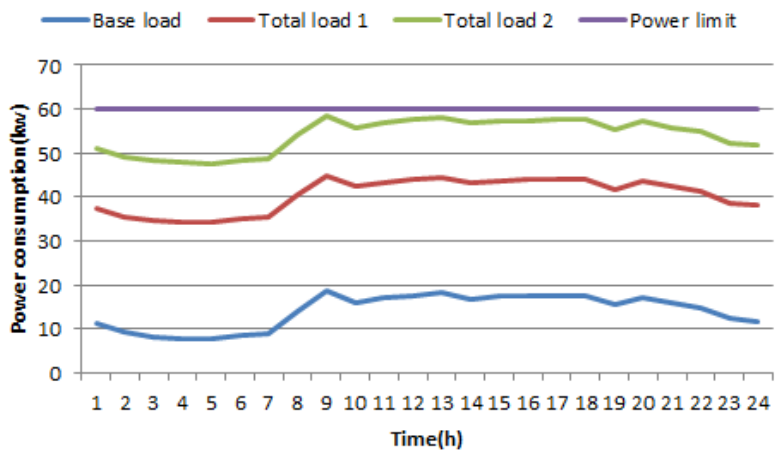

Fig. 3 Total power consumption for the network.

Table 1 EV specification.

\begin{tabular}{lll}
\hline Model & $\begin{array}{l}\text { Battery } \\
\text { capacity }(\mathrm{kWh})\end{array}$ & $\begin{array}{l}\text { Rated charging } \\
\text { power }(\mathrm{kW})\end{array}$ \\
\hline Tesla Model S EV & 60 & 10 \\
Honda Fit EV & 20 & 6.6 \\
\hline
\end{tabular}

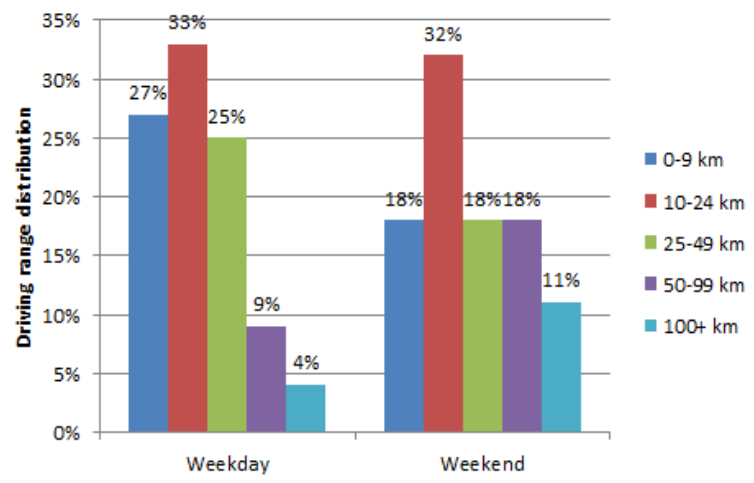

Fig. 4 Driving range distributions.

Fig. 4 demonstrates the customers driving range distributions. We can see that most people will commute within $50 \mathrm{~km}$. There will be a higher probability for longer range driving in the weekend. 
Table 2 Customers' departure time distribution.

\begin{tabular}{lllll}
\hline Hours & $<7$ a.m. & $\begin{array}{l}7 \text { a.m.- } \\
9 \text { a.m. }\end{array}$ & $\begin{array}{l}9 \text { a.m.- } \\
12 \text { p.m. }\end{array}$ & $>12$ p.m. \\
\hline $\begin{array}{l}\text { Percentage } \\
(\%)\end{array}$ & 18 & 70 & 3 & 5 \\
\hline
\end{tabular}

Table 3 Customers' arrival time distribution.

\begin{tabular}{lllllll}
\hline Hours & $<12$ p.m. & 12 p.m.- & 2 p.m.- & 5 p.m.- & 7 p.m.- & 2 \\
& & & 5 p.m.m. & 7 p.m. & 9 p.m. & p.m. \\
\hline $\begin{array}{l}\text { Percentage } \\
(\%)\end{array}$ & 3 & 2 & 31 & 53 & 7 & 2 \\
\hline
\end{tabular}

This is probably for shopping and pleasant during the weekend. The departure and arrival time for the EV owners are shown in Tables 2 and 3, respectively. Seventy percent of all the drivers will leave home between 7 a.m. and 9 a.m. More than half of the people will arrive between 5 p.m. and 7 p.m.

For charging pricing, some utility companies adopt the TOU (time-of-use) electricity pricing structure to encourage customers to shift their power consumption to valley hours. In this pricing structure, the electricity price will be high for the peak hours of power consumption and low for valley hours. We also utilize the TOU pricing structure in our control framework.

\subsection{Customer Satisfaction Model}

Most of previous papers discuss the EV charging control strategies to minimize the aggregated charging cost or mitigate the negative impacts on power system, with little work discussing the user satisfactions. In order to compare user satisfaction for different charging control algorithms, we propose a new user satisfaction model. We observe three determining factors: the user controllability of the charging, the normalized final BSOC (battery state of charge) after the charging and the relative total charging cost.

$$
S=S I\left(\mathrm{BSOC}_{\text {final }} / \mathrm{BSOC}_{\text {target }}\right)\left(\mathrm{C}_{\text {un }} / \mathrm{C}_{\text {real }}\right)
$$

Eq. (1) shows our proposed model for the calculation of user satisfaction $S$, which is the product of the three factors; SI denotes the satisfaction index. For those people who can control the EV charging, SI is set as 1; otherwise it is a constant positive fractional number; $\mathrm{BSOC}_{\text {final }}$ denotes the final BSOC after the charging; $\mathrm{BSOC}_{\text {target }}$ is the required $\mathrm{BSOC}$ predefined by the customer. The ratio of these two gives the indication of the battery charging status. Finally, $C_{u n}$ is the charging cost for EV charging without any control methods; and $C_{\text {real }}$ shows the charging cost with certain EV charging control algorithm.

The user satisfaction for a specific network is defined in Eq. (2), which is the average among all the customers. In the equation, $n$ means the number of $\mathrm{EV}$ customers in the neighborhood level network. $S_{i}$ shows the customer satisfaction value for $i$-th customer.

$$
S_{\text {average }}=\sum_{i=1}^{n} S_{i} / n
$$

\section{Methodology}

As mentioned in Section 2, we consider that, there are two groups of customers in a neighborhood level network: contractual customers and non-contractual customers. It is assumed that, the EV charging control and communication devices have been installed for all the houses in the given neighborhood level network for contractual customers. The system operator can access full information of the contractual EVs: the real time BSOC, arrival time, predefined departure time and final BSOC requirement for their EVs. The charging behaviors of all the contractual EVs will be controlled by the system operator. For those non-contractual EVs, there is less information exchange between the EVs and the system operator. But the system operator can access the charging histories of these EVs. The non-contractual customers are assumed to be rational and would follow the electricity price signal to arrange the EV charging.

\subsection{Mixed Control Algorithm}

Fig. 5 shows the overview of the proposed energy management system. It is assumed that, there is a system operator who is responsible for controlling the charging for all the contractual EVs and deciding the time-of-use price structure for the neighborhood level network. 


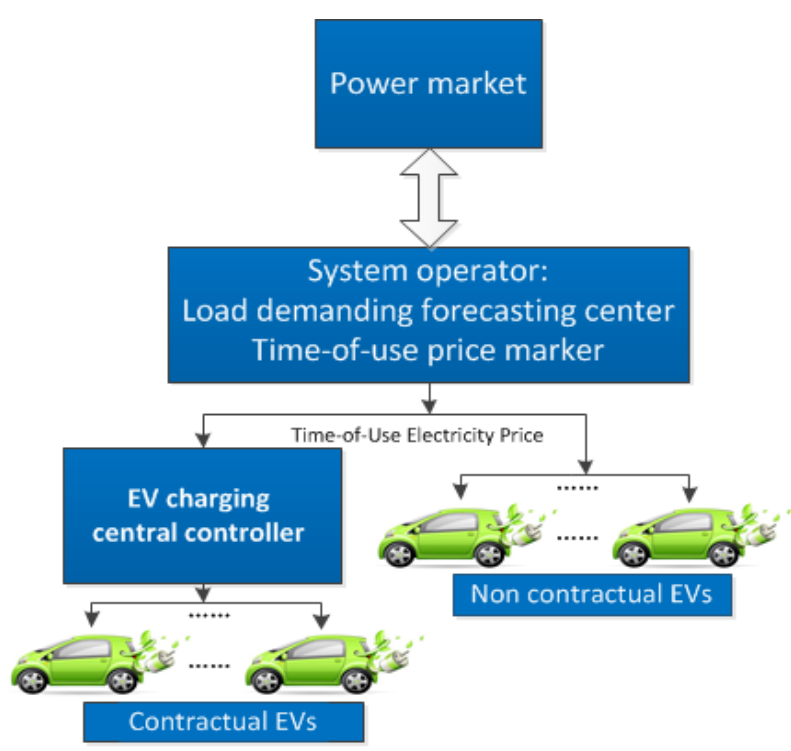

Fig. 5 Energy management system with mixed control strategy.

There are mainly three steps for the proposed mixed control algorithm. The first step is the load forecasting for base load power consumption. Different from Ref. [24], in this paper, we focus on the forecasting for base load power consumption. Load forecasting has been discussed in several previous Refs. [25-28]. In this paper, the time slot is chosen as $1 \mathrm{~h}$. Weather information, weekday/weekend classification and hourly power consumptions for the previous day are used for load forecasting. Neural network is used for load forecasting for every hour in the next $24 \mathrm{~h}$.

The second step is to determine the time-of-use price structure based on the forecasted load consumption. The pricing model proposed in Ref. [19] is adopted to determine the TOU structure. Fig. 6 shows an example the TOU price structure. We first find the maximal and minimal load consumption based on the forecasted result. The obtained timing region will be partitioned into three zones: valley, medium and peak hours. We assign $0.112 \$ / \mathrm{kWh}$, $0.135 \$ / \mathrm{kWh}$ and $0.072 \$ / \mathrm{kWh}$ for the medium hours, peak hours and valley hours. The TOU price signal will be informed to all the non-contractual customers in the third step. TOU price acts as an incentive to realize the decentralized control for those non-contractual customers.

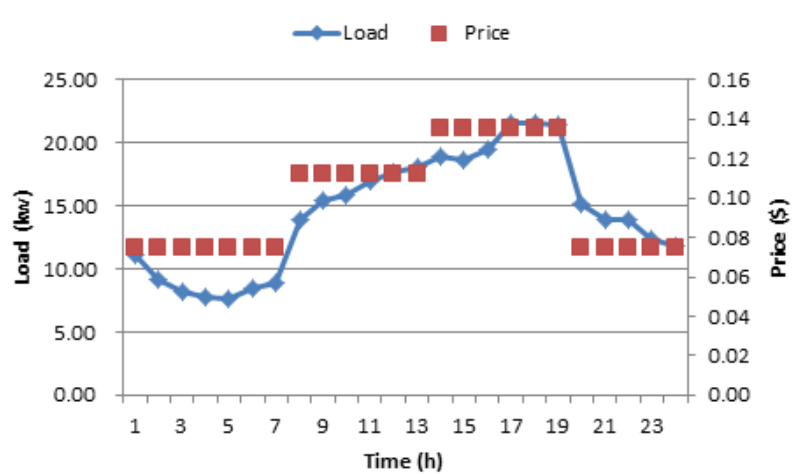

Fig. 6 Identification of zones for pricing.

\subsection{Objective Function}

The objective for our proposed optimization framework is to minimize the total charging cost for the contractual customers. The objective function $F$ is shown in Eq. (3):

$$
F=\min \sum_{i=1}^{m} \sum_{t} c_{t} \cdot P_{i, t}
$$

In total, we assume there are $n$ customers and $m$ of all the customers are willing to give out the EV charging control. Without loss of generality, we assume that each customer owns one electric vehicle. Then there are $m$ electric vehicles owned by contractual customers in the neighborhood level network, and $n-m$ EVs owned by non-contractual customers. $P_{i, t}$ means the charging power for the $i$-th $\mathrm{EV}$ in time interval $t$ and $c_{t}$ is the electricity price for that time interval.

\subsection{Constraints}

For the stability of the power system, all the following constraints should be satisfied in all time slots.

Total power constraint is shown in Eq. (4). It means that, for every time interval, the total charging power including the base load power consumption and the EV charging power consumption should be less than a certain value predefined by the transformer specification. As mentioned in Eq. (4), $P B_{i, t}$ means the base load consumption in $i$-th home for $t$-th time interval, $P C_{i}, t$ describes the charging power 
consumption, and $P T_{\max }$ means the upper limit for the total power consumption.

$$
0 \leq \sum_{i=1}^{n}\left(P B_{i, t}+P C_{i, t}\right) \leq P T_{\max }
$$

EV charging power consumption is shown in Eq. (5). Continuous EV charging is adopted in this paper, which means that, the EV can be charged with power from zero to $P C_{\max }$, the maximal rated $\mathrm{EV}$ charging power.

$$
0 \leq P C_{i, t} \leq P C_{\max }
$$

BSOC constraints are shown in Eqs. (6) and (7). Eq. (6) shows that in all time slots the EV BSOC should be smaller than a predefined value $\left(\mathrm{BSOC}_{\max }\right)$. Eq. (7) means that after the charging period, the final $\mathrm{EV}$ BSOC $\left(\mathrm{BSOC}_{\text {final }}\right)$ should satisfy the user predefined $\mathrm{BSOC}\left(\mathrm{BSOC}_{\text {predefined }}\right)$ requirement.

$$
\begin{gathered}
0 \leq \mathrm{BSOC}_{i, t} \leq \mathrm{BSOC}_{\max } \\
B S O C_{\text {predefined }} \leq B S O C_{\text {final }} \leq B S O C_{\text {max }}
\end{gathered}
$$

Eq. (8) shows the BSOC updating constraint. Cap in this equation means the battery capacity. $C$ is determined by the length of time interval, if time interval is $1 \mathrm{~h}, C$ will be $1 . \delta$ shows the conversion efficiency for $\mathrm{EV}$ charging.

$$
\mathrm{BSOC}_{t+1}=\mathrm{BSOC}_{t}+C \cdot\left(\delta \cdot P C_{i} / \mathrm{Cap}\right) \times 100
$$

\section{Simulation Results}

\subsection{Simulation Setup}

The proposed EV charging control framework is tested with real data for a neighborhood level network from a local utility company. There are in total seven homes in the network as shown in Fig. 1. Uncontrolled charging and centralized control EV charging are used as comparisons for the proposed mixed EV charging control framework. For uncontrolled EV charging, we assume that, the customers will start charging their EVs once they arrive home with rated charging power. For centralized charging control, we assume that, the system controller can control the EV charging directly for all the EVs in the network. Simulation results for charging cost, user satisfaction, maximal EV penetration and peak power reduction are analyzed.

As shown in Table. 4, we consider three cases for the mixed charging control strategy according to the number of contractual customers. For example, in Case 2, four homes out of all the homes are contractual customers. This is also defined as the basic case for mixed control strategy.

Two kinds of electric vehicles: Honda Fit and Tesla model $\mathrm{S}$ are considered. The specifications for these two EVs are shown in Table. 1. The arrival BSOC, arrival time and departure time for all these EVs will be set according to the probability distribution of the survey mentioned in Section 2. For the total power constraints, $P T_{\max }$ is set as 60 , the $\mathrm{BSOC}_{\max }$ is set as $95, \mathrm{BSOC}_{\text {pre }}$ is set as 80 . The average initial BSOC for all EVs is $45 \%$. For non-contractual customers, the user satisfaction index $S I$ is set to be 0.8 . The conversion efficiency for EV charging is set as 0.95 . The optimization problem is solved with CPLEX (simplex method as implemented in the $\mathrm{C}$ Programming Language) 12.0 .

\subsection{Charging Cost and User Satisfaction Result}

As described in Section 3, we assume that, the total operation cost and user satisfaction are two major factors for neighborhood level customers. The charging costs and user satisfactions for three representative charging control strategies are shown in Figs. 7 and 8.

The charging cost for three control strategies are shown in Fig. 7. We can see that, the charging cost for the mixed charging control strategy is significantly less than the uncontrolled strategy and close to the centralized control strategies. With mixed control strategy the charging cost will be reduced $16.2 \%$ and 19.5\% for Honda Fit and Tesla Model S compared to uncontrolled EV charging. 
Table 4 Mixed control strategy cases.

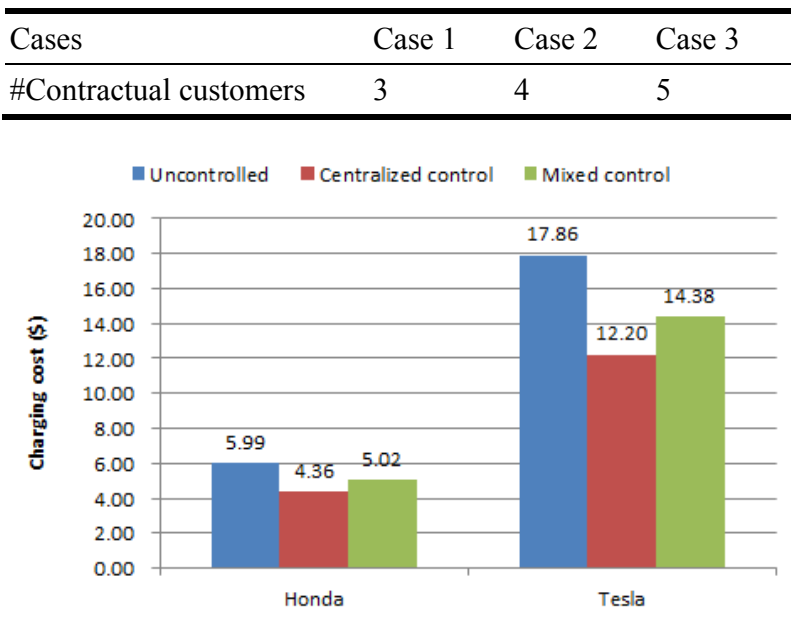

Fig. 7 Charging cost for different control strategies.

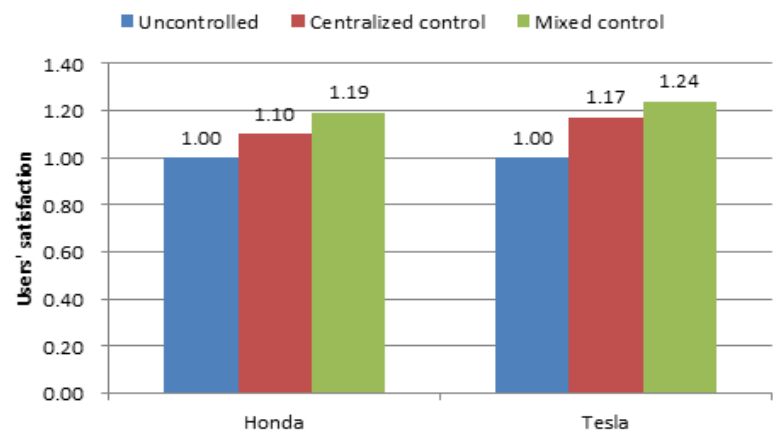

Fig. 8 User satisfaction for different control strategies.

User satisfaction for three representative charging control strategies is illustrated in Fig. 8. It can be seen that, for the mixed strategy, customers can have the highest overall user satisfactions. This is because the non-contractual customers can get full control of the EV charging and the overall charging cost is reduced. We can see that with the proposed criteria, user satisfaction can be well analyzed for different control algorithms.

The charging costs for the three cases for mixed control strategy are listed in Table. 5. We can see that, with the increasing of the number of contractual customers, we will have a lower charging cost.

\subsection{Peak Power Consumption}

Peak power consumption is another important factor for utility companies, as the overloaded power consumption will accelerate the grid ageing [29]. As mentioned in Ref. [30], a higher peak demand will require a larger generation capacity and cause the power system to be unstable. Fig. 9 shows the peak power consumptions for different control strategies. As the centralized control strategy could control the charging of all the EVs, this control algorithm provides the lowest peak power consumption. It can also be seen that, with the proposed mixed control strategy, the peak power consumption could be reduced significantly. Compared with uncontrolled EV charging, the peak power consumptions are reduced $37.6 \%$ for Honda Fit and $33.22 \%$ for Tesla Model S.

\subsection{EV Penetration Result}

The adoption of the EVs will increase the burden on the power system which may require related infrastructure upgrade. It would be an interesting question for the utility companies to know the potential EV penetration for a given neighborhood level network. The possible EV penetration for a given network is discussed in Refs. [5, 31]. However, most of them focused on pure centralized or decentralized control strategy. This may not be ideal or realistic as discussed in previous sections.

The proposed mixed control framework can also be adopted to find a more realistic EV penetration for a

Table 5 Contractual Customer number.

\begin{tabular}{llll}
\hline \# Contractual customers & 3 & 4 & 5 \\
\hline Charging cost Honda (\$) & 5.33 & 5.02 & 4.9 \\
Charging cost Tesla (\$) & 15.41 & 14.38 & 13.7 \\
\hline
\end{tabular}

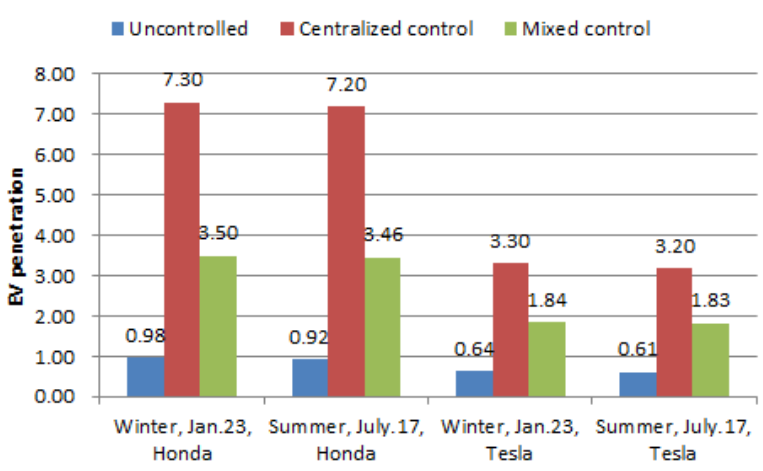

Fig. 9 Peak power consumption. 
given network. To find the EV penetration, we can set the objective function in Section 4 as the number of EVs in every home. With the proposed framework, the simulation result will be more practical.

The EV penetration for three representative charging control strategies is analyzed on the two specific days. Simulation results are shown in Fig. 10. As the base load consumption is higher in summer than in winter, the EV penetration in winter is a little higher than that in summer. In all three strategies, we can get the highest EV penetration with the centralized control strategy.

For typical summer day, EV penetration can only be 0.92 for Honda Fit and 0.61 for Tesla Model S if the EV charging is left uncontrolled. With mixed charging control strategy, the EV penetration could be 3.46 for Honda Fit EV and 1.83 for Tesla Model S. We can also see that as the Tesla Model S require high EV charging rate which limits its penetration potential.

EV penetrations for mixed control strategy with different contractual customer numbers are shown in Fig. 11. We can see that, with an increase of contractual customers, the EV penetration will increase accordingly.

\section{Conclusions}

The fast development of EVs will induce negative impacts for power system and accelerate grid ageing if the EV charging is left uncontrolled. In this paper, the charging control for the EVs has been analyzed. A mixed control strategy and its optimization framework are proposed to mitigate the EV charging problem while considering the willingness of the customers. Compared with previous charging control strategies, in the proposed framework we consider that in the actual neighborhood level network, not all the customers would like to sign related contract with the utility companies and give out the charging control for their EVs.

A new user satisfaction model is also described in

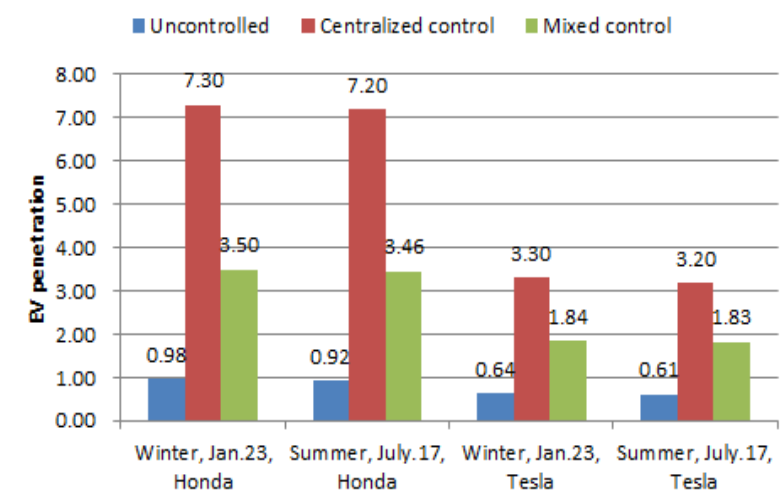

Fig. 10 EV penetration for different control strategies.

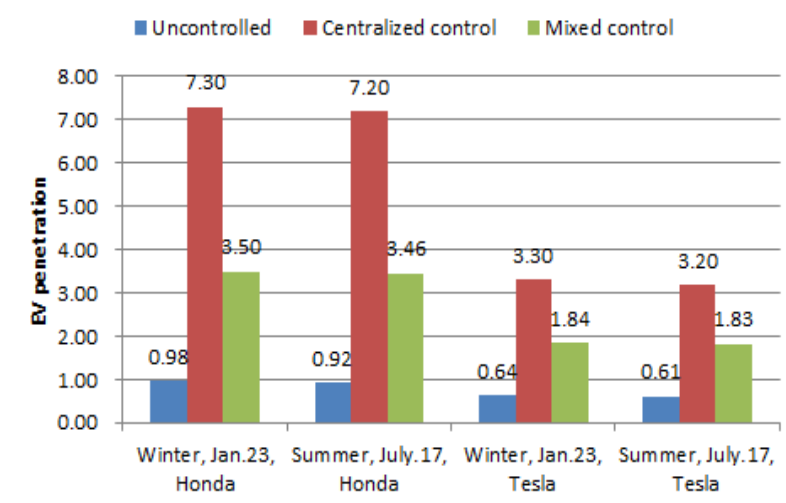

Fig. 11 EV penetration for mixed control strategies.

this paper, which will help study the user satisfactions for different control strategies. The EV penetration, charging cost and user satisfactions for the proposed control framework, as well as the centralized control strategy and uncontrolled charging are studied. Simulation results show the efficiency of the proposed smart charging control framework. With the proposed method, we can get a higher EV penetration and lower charging cost. We can see that, the proposed strategy is beneficial for both the utility companies and the customers.

\section{References}

[1] DOE (US Department of Energy). 2011. One Million Electric Vehicles by 2015. Virginia: US Department of Energy.

[2] Shireen, M. K., and Das, D. 2008. "Biohydrogen as a Renewable Energy Resource-Prospects and Potentials." International Journal of Hydrogen Energy 33 (1): 258-63.

[3] Green, A. R. C., Wang, II, L., and Alam, M. 2011. "The 
Impact of Plug-in Hybrid Electric Vehicles on Neighborhood Networks: A Review and Outlook." Renew. Sustain. Energy Rev. 15 (1): 544-53.

[4] Azad, A. P., Olivier, B., and Pfeiffer, L. 2013. "An Optimal Control Approach for EV Charging with Distribution Grid Ageing." In Proceedings of the 2013 First International Black Sea Conference on Communications and Networking (BlackSeaCom), IEEE, 206-10.

[5] Peter, R., Damian, F., and Andrew, K. 2012. "Optimal Charging of Electric Vehicles in Low-Voltage Distribution Systems." IEEE Transactions on Power Systems 27 (1): 268-79.

[6] Rotering, N., and Ilic, M. 2011. "Optimal Charge Control of Plug-in Hybrid Electric Vehicles in Deregulated Electricity Markets.” IEEE Trans. Power Systems 26 (3): 1021-9.

[7] Ahn, C., Li, C. T., and Peng, H. 2011. "Decentralized Charging Algorithm for Electrified Vehicles Connected to Smart Grid." In Proceedings of the ACC (American Control Conference), 3924-9.

[8] Li, N., Chen, L., and Low, S. H. 2011. "Optimal Demand Response Based on Utility Maximization in Power Networks." In Proceedings of the IEEE Power and Energy Society General Meeting, 1-8.

[9] Kahn, M. E. 2008. "Green Market Geography: The Spatial Clustering of Hybrid Vehicle and LEED Registered Buildings." Working Paper 2008-19, UCLA (University of California Los Angeles) Ziman Center for Real Estate.

[10] Aultman-Hall, L., Sears, J., Dowds, J., and Hines, P. 2012. "Travel Demand and Charging Capacity for Electric Vehicles in Rural States: A Vermont Case Study." Presented at the Transport Research Board 92nd Annual Meeting, Washington, USA.

[11] Kempton, W., and Letendre, S. E. 1997. "Electric Vehicles as a New Power Source for Electric Utilities." Transportation Research Part D: Transport and Environment 2 (3): 157-75.

[12] Chagngsun, C. H. 2011. "Dencentralized Charging Alogorithm for Electricfied Vehicles Connected to Smart Grid." In Proceedings of the ACC (American Control Conference), 3924-9.

[13] Komang A. I., and Kab, S. 2013. "A Centralized Scheme Based on User Satisfaction Fairness and Cost." In Proceedings of the IEEE ISGT Asia (Innovative Smart Grid Technologies-Asia), 1-4.

[14] Papadaskalopoulos, D., and Strbac, G. 2012. "Decentralized Participation of Electric Vehicles in Network-Constrained Market Operation." In Proceedings of the 3rd IEEE PES International Conference and Exhibition on ISGT (Innovative Smart Grid Technologies)
Europe, 1-8.

[15] Xiaomin, X., and Sioshansi, R. 2014. "Using Price-Based Signals to Control Plug-in Electric Vehicle Fleet Charging." IEEE Transaction on Smart Grid 5 (3): 1451-64.

[16] Hong, L., and Shaoyun, G. 2013. "Optimization of TOU Price of Electricity Based on Electric Vehicle Orderly Charge." In Proceedings of the IEEE PES (Power and Energy Society) General Meeting, 1-5.

[17] Vaya, M. G., and Anderson, G. 2012. "Centralized and Decentralized Approaches to Smart Charging of Plug-in Vehicles." In Proceedings of the IEEE Power and Energy Society General Meetings, 1-8.

[18] Ruoyang, L., Qiuwei, W., and Oren, S. S. 2014. "Distributional Locational Marginal Pricing for Optimal Electric Vehicle Charging Management." IEEE Trans. Power Systems 29 (1): 203-11.

[19] Yan, Q. 2014. "A Multi-tied Real-Time Pricing Algorithm for Electric Vehicle Charging Station." In Proceedings of the ITEC (IEEE Transportation Electrification Conference) and Expo, 1-6.

[20] Grunewald, A., Hardt, S., and Mielke, M. 2012. "A Decentralized Charge Management for Electric Vehicles Using a Genetic Algorithm: Case Study and Proof-of-Concept in Java and FPGA." In Proceedings of the IEEE CEC (Congress on Evolutionary Computation), 1-7.

[21] TESLA. 2014. "85 kWh Battery." Tesla Motors. Accessed May 20, 2015. http://www.teslamotors.com/en_CA/charging\#/basics.

[22] HONDA. 2014. "2014 Accord Plug-in.” American Honda Motor Co., Inc. Accessed May 20, 2015. http://automobiles.honda.com/accord-plug-in/charging-an -electric-vehicle.aspx.

[23] Oliver, B., Probe, P., and Flores, M. 2013. "EMAP: Informing the Development of an EV Deployment Strategy in the City of Ottawa." Presented at Evve Conference and Trade Show, Gatineau, Canada. Accessed May 20, 2015. http://emc-mec.ca/evve2013/ pdfs/22OCT/TS8/3_Oliver_Flores.pdf.

[24] Wu, D., Haibo, Z., and Benoit, B. 2014. "Neighborhood Level Network Aware Electric Vehicle Charging Management with Mixed Control Strategy." In Proceedings of the 2014 IEEE IEVC (International Electric Vehicle Conference), 1-7.

[25] Dongxiao, N., Yongli, W., and Desheng, D. W. 2010. "Power Load Forecasting Using Support Vector Machine and ant Colony Optimization." Expert Systems with Applications 37 (3): 2531-9.

[26] Bakirtzis, A. G. 1996. "A Neural Network Short Term Load Forecasting Model for the Greek Power System." IEEE Transactions on Power Systems 11 (2): 858-63. 
[27] Senjyu, T. 2002. "One-Hour-Ahead Load Forecasting Using Neural Network." IEEE Transactions on Power Systems 17 (1): 113-8.

[28] Lu, C. N., Wu, H. T., and Vemuri, S. 1993. "Neural Network Based Short Term Load Forecasting." IEEE Transactions on Power Systems 8 (1): 336-42.

[29] Rutherford, M. J., and Yousefzadeh, V. 2011. "The Impact of Electric Vehicle Battery Charging on Distribution Transformers." In Proceedings of the 26th
IEEE APEC (Applied Power Electronics Conference) and Exposition, 396-400.

[30] Shizhen, Z., Xiaojun, L., and Minghua, C. 2013. "Peak-Minimizing Online EV Charging." Allerton.

[31] Rocha, A. P. M., Peças, L. J. A., and Soares, F. J. 2011. "Electric Vehicles Participating in Frequency Control: Operating Islanded Systems with Large Penetration of Renewable Power Sources." In Proceedings of the IEEE Power Tech., 1-6. 